\title{
Acute and Long-Term Effects of Noise Exposure on the Neuronal Spontaneous Activity in Cochlear Nucleus and Inferior Colliculus Brain Slices
}

\author{
Moritz Gröschel, Jana Ryll, Romy Götze, Arne Ernst, and Dietmar Basta \\ Department of Otolaryngology at Unfallkrankenhaus Berlin, Charité Medical School, Warener Straße 7, 12683 Berlin, Germany \\ Correspondence should be addressed to Dietmar Basta; dietmar.basta@rz.hu-berlin.de
}

Received 19 February 2014; Revised 20 June 2014; Accepted 20 June 2014; Published 8 July 2014

Academic Editor: Claus-Peter Richter

Copyright (C) 2014 Moritz Gröschel et al. This is an open access article distributed under the Creative Commons Attribution License, which permits unrestricted use, distribution, and reproduction in any medium, provided the original work is properly cited.

\begin{abstract}
Noise exposure leads to an immediate hearing loss and is followed by a long-lasting permanent threshold shift, accompanied by changes of cellular properties within the central auditory pathway. Electrophysiological recordings have demonstrated an upregulation of spontaneous neuronal activity. It is still discussed if the observed effects are related to changes of peripheral input or evoked within the central auditory system. The present study should describe the intrinsic temporal patterns of single-unit activity upon noise-induced hearing loss of the dorsal and ventral cochlear nucleus (DCN and VCN) and the inferior colliculus (IC) in adult mouse brain slices. Recordings showed a slight, but significant, elevation in spontaneous firing rates in DCN and VCN immediately after noise trauma, whereas no differences were found in IC. One week postexposure, neuronal responses remained unchanged compared to controls. At 14 days after noise trauma, intrinsic long-term hyperactivity in brain slices of the DCN and the IC was detected for the first time. Therefore, increase in spontaneous activity seems to develop within the period of two weeks, but not before day 7. The results give insight into the complex temporal neurophysiological alterations after noise trauma, leading to a better understanding of central mechanisms in noise-induced hearing loss.
\end{abstract}

\section{Introduction}

Long-lasting noise exposure at high intensities leads to an immediate posttraumatic temporary shift of hearing thresholds (TTS) and is followed by a long-lasting permanent threshold shift (PTS) if sensory tissue is damaged to a large extent at high sound intensities $[1,2]$. It has already been shown that PTS is accompanied by dramatic changes of cellular properties within the central auditory pathway, such as neuronal shrinkage, axonal and synaptic degeneration [3$6]$, changes in synaptic activity $[7,8]$, and a decrease in neuronal cell density $[9,10]$.

Electrophysiological research has shown that spontaneous neuronal activity and compound action potentials are decreased in the auditory nerve after noise exposure [11, 12]. Similar findings have been reported within the first days postexposure in the central structure of the dorsal cochlear nucleus $[13,14]$. However, long-lasting effects of noise trauma are an upregulation of spontaneous firing rates in several structures of the central auditory pathway [14-16] and an increase in excitability, whereby excitatory thresholds are elevated [17]. These changes seem to be based upon both a reduction of GABAergic inhibition and an enhancement of excitation within the impaired structures $[18,19]$.

It is still a matter of debate if the observed effects are related to the noise-induced changes of afferent peripheral input or directly evoked by an overstimulation of the entire auditory pathway. It seems that central auditory structures contribute to the development of an acute auditory threshold shift since it has been shown that some central effects of noise could be short-term only $[10,20]$. Furtherly, clinical sequalae of noise exposure, such as tinnitus and hyperacusis, are frequently present after the hearing loss has recovered which cannot be fully explained with cochlear pathology [21]. 
These discrepancies are possibly related to additional, central mechanisms involved in the generation of noise-induced hearing loss (NIHL). To date, only a few studies focused on the time course of the electrophysiological changes after noise trauma. However, in vivo findings indicate that hyperactivity in the central auditory system develops during the first days after trauma [22], whereby the DCN seems to play a key role in maintaining the increased neuronal firing throughout the ascending pathway $[23,24]$.

Electrophysiological changes in the central auditory system have been commonly studied in vivo (anaesthetized animals). Thereby, it is hardly possible to differentiate if changes in central auditory processing rely on altered central auditory structures under observation or if they were influenced by interacting ascending and descending neuronal projections. By using electrophysiological recording techniques in brain slices, intrinsic cellular changes of different levels of central auditory processing could be evidenced. With this methodology, single brain structures disconnected from peripheral and central afferent and efferent input can be investigated in detail.

The aim of the present study was therefore to describe the temporal patterns of spontaneous, neuronal single-unit activity upon noise-induced hearing loss in different key structures of the lower central auditory pathway. This experimental procedure allows distinguishing between acute and long-term changes in the dorsal and ventral cochlear nucleus as well as the inferior colliculus.

\section{Methods}

2.1. Noise Exposure. 57 normal hearing adult mice (NMRIstrain, age 1 to 3 months) of both sexes were investigated. The experimental protocol was approved by the governmental commission for animal studies (LaGeSo Berlin, approval number: G 0416/10). Experiments were carried out in accordance with the EU Directive 2010/63/EU on the protection of animals used for scientific purposes. All efforts were made to minimize pain or discomfort.

The animals were anaesthetized for $3 \mathrm{~h}$ with ketamine/ xylazine $(60 \mathrm{mg} / \mathrm{kg}$ ketamine, $6 \mathrm{mg} / \mathrm{kg}$ xylazine). 32 mice were exposed to noise during the anaesthesia in a soundproof chamber $(0.8 \mathrm{~m} \times 0.8 \mathrm{~m} \times 0.8 \mathrm{~m}$, minimal attenuation $60 \mathrm{~dB}$ SPL) with a broad-band white noise $(5-20 \mathrm{kHz})$ at $115 \mathrm{~dB}$ SPL. Noise was delivered binaurally by high tone loudspeakers (HTC 11.19, Visaton, Haan, Germany) placed above the animal's head. Speakers were connected with an audio amplifier (Tangent AMP-50, Aulum, Denmark) and a DVD Player (DK DVD-438, Ratingen, Germany). Soundpressure level (SPL) was calibrated by using a sound level meter (Voltcraft 329, Hirschau, Germany) placed close to the animal's ear. Anaesthesia was controlled by a video camera inside of the lighted chamber. Body temperature was maintained at a constant level of $37^{\circ} \mathrm{C}$. Mice were investigated immediately after the noise exposure (acute group, $n=9$ ) or were kept in their cages for one (7-day group, $n=17$ ) or two (14-day group, $n=6$ ) weeks to be investigated seven or fourteen days later. Unexposed animals were used as controls (control group, $n=25$ ).
2.2. Electrophysiological Recordings. Extracellular single-unit recordings were performed in acute living brain slices to investigate the spontaneous firing rates at different stages after noise trauma (acute group, $n=9$; 7-day group, $n=17$; 14-day group, $n=6$ ) compared to unexposed controls $(n=25)$. Therefore, the animal (only one animal was investigated at each experimental day) was decapitated and the brain was carefully taken out. Using a vibrating microtome (Model 1000 plus, Vibratome, St. Louis, Missouri, USA), $300 \mu \mathrm{m}$ thick frontal slices (including CN or IC, resp.) were microdissected. After $2 \mathrm{~h}$ of incubation in carbogenized $\left(95 \% \mathrm{O}_{2}-5 \% \mathrm{CO}_{2}\right)$ artificial cerebrospinal fluid (cACSF) at $35^{\circ} \mathrm{C}$ under submerged conditions, one slice was transferred to a submerged-type recording chamber. The recording chamber was continuously perfused $(2.5 \mathrm{~mL} / \mathrm{min})$ with warm $\left(36^{\circ} \mathrm{C}\right)$ cACSF. The cACSF contained the following concentrations (in $\mathrm{mM}$ ): $124 \mathrm{NaCl}$, $3 \mathrm{KCl}, 1.25 \mathrm{NaH}_{2} \mathrm{PO}_{4}, 1.8 \mathrm{MgSO}_{4}, 1.6 \mathrm{CaCl}_{2}, 10$ glucose, and $26 \mathrm{NaHCO}_{3}$. The temperature of the bath solution was measured with a thermistor probe in the recording chamber and regulated within a small range by a temperature controller (SCTC-20E, npi-electronics, London, UK). Action potentials from spontaneously active neurons within the dorsal and ventral cochlear nucleus (DCN and VCN, resp.) and the inferior colliculus (IC) (localized using the stereotaxic brain atlas from Paxinos and Franklin [25], Figure 1) were recorded with glass electrodes (GB120-F10, Science Products, Hofheim, Germany). The electrodes were pulled on a P97 horizontal puller (Sutter Instruments, Novato, CA, USA) and back-filled with a sodium chloride solution $(154 \mathrm{mM})$. The resulting electrode resistance was approximately $2 \mathrm{M} \Omega$. The signal was amplified $(10,000 \mathrm{x})$ and filtered $(0.3-20 \mathrm{kHz}$ band pass) (Model 1800, A-M Systems Inc., Sequim, Washington, USA), visualized on an oscilloscope, digitized by a 1401 Plus interface (Cambridge Electronic Design Ltd., Cambridge, UK), and stored in the Spike2 software format (Cambridge Electronic Design Ltd., Cambridge, UK). Spikes were detected offline by using the Spike2 software. After establishing a stable recording, neuronal spontaneous activity was measured for $3 \mathrm{~min}$ utes.

2.3. Statistical Procedures. The resulting mean neuronal spontaneous activities (events per second) of the experimental groups were compared with the controls for each brain region by the $U$-test as the data were not normally distributed. Moreover, each investigated structure was topographically subdivided into a high- and low-frequency area. Data of these subdivisions have been compared within each structure as well as between frequency-related substructures of different experimental groups using $U$-test (not normally distributed data) or $t$-test (normally distributed data). Data distribution was tested by applying the Kolmogoroff-Smirnoff test. The software SPSS (IBM SPSS Statistics Version 20, IBM Corp., Armonk, New York, USA) was used for all statistical analyses. The level of significance was $P<0.05$. A Bonferroni correction was applied for multiple comparisons. 

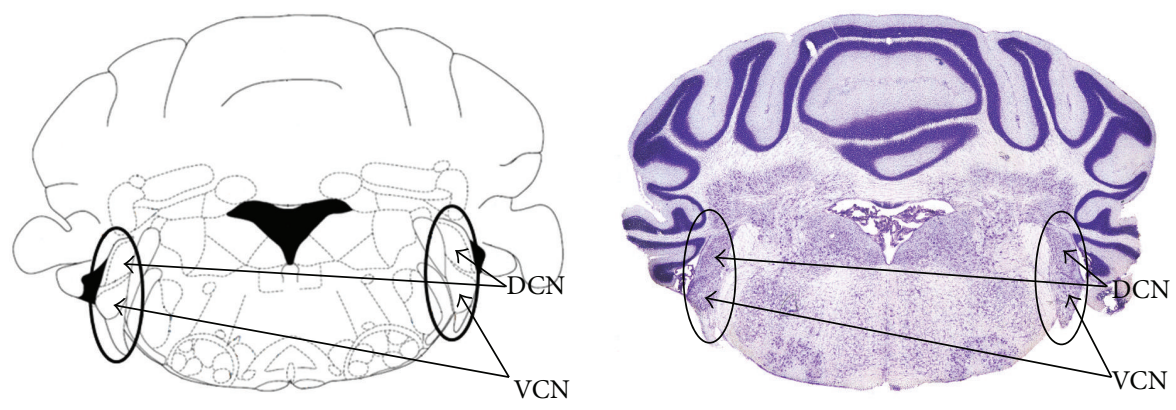

(a)
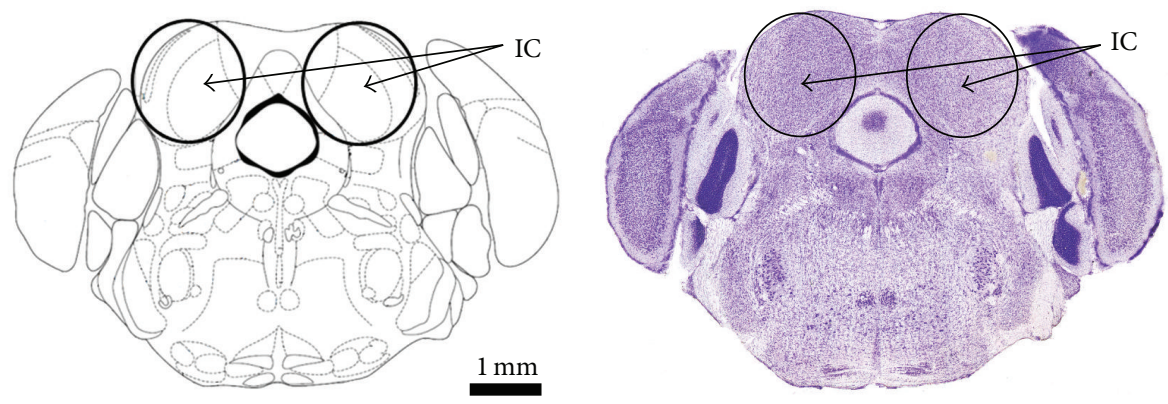

(b)

FiguRE 1: Transversal schematic diagrams (left) and corresponding histological sections (Nissl-stained $40 \mu \mathrm{m}$ brain slice preparations, right) from the mouse brain including the investigated structures of the cochlear nucleus (black labelled area in (a)) in the brainstem with its dorsal and ventral subdivision (DCN and VCN, resp.) and the inferior colliculus (IC, black labelled area in (b)) in the midbrain. Layers correspond to the prepared brain slice sections. Pictures were taken and modified from the mouse brain atlas by Paxinos and Franklin [25].

\section{Results}

3.1. Cochlear Nucleus. The extracellular electrophysiological recordings in brain slices showed significant changes in spontaneous activity immediately as well as two weeks after noise exposure compared to controls. Interestingly, spontaneous neuronal firing rates did not show significant differences at day 7 after trauma. An example of two DCN neurons spike trains from a control and a 14-day group animal is given in Figure 2. An increase in spontaneous firing in the noiseexposed group two weeks after trauma is represented by the higher spike rate of the 14-day group neuron (Figure 2).

The mean rate of action potentials in the recorded DCN units was increased significantly from $8.8 \pm 1.4 \mathrm{imp} / \mathrm{s}$ (95 recorded neurons) in the control group to $12.6 \pm 1.8 \mathrm{imp} / \mathrm{s}(13$ recorded neurons) acutely after noise exposure $(P=0.002$; Figure 3). A similar observation has been made in the VCN of the acute group, where the mean spontaneous firing rate was $14.2 \pm 1.8 \mathrm{imp} / \mathrm{s}$ ( 24 recorded neurons) compared to $10.6 \pm 2.1 \mathrm{imp} / \mathrm{s}$ (53 recorded neurons) in the control group $(P=0.001$; Figure 4$)$. Changes in spontaneous neuronal firing rates were not significantly different in the 7-day group when compared to controls. This holds true for the DCN (7 days: $11.5 \pm 1.6 \mathrm{imp} / \mathrm{s}$ (124 recorded neurons), $P=0.131$ ) and the VCN (7 days: $9.2 \pm 2.1 \mathrm{imp} / \mathrm{s}$ (43 recorded neurons), $P=0.979$ ).
When analyzing the 14-day group data, it became evident that significant differences were present in the DCN, but not in the VCN compared to controls. In the DCN, mean spontaneous activity was raised to $33.3 \pm 6.3 \mathrm{imp} / \mathrm{s}$ (44 recorded neurons, $P=0.001$; Figure 3 ) and was not statistically significantly elevated in the VCN $(11.0 \pm 1.2 \mathrm{imp} / \mathrm{s}$ (38 recorded neurons), $P=0.019$; Figure 4 ).

3.2. Inferior Colliculus. The spontaneous activity in the IC immediately after the noise trauma was not significantly different to controls (control group: $4.9 \pm 0.4$ (56 recorded neurons), acute group: $5.9 \pm 0.5$ (40 recorded neurons), $P=$ 0.55; Figure 5).

Changes in spontaneous neuronal firing rates were also not significantly different in the 7-day group when compared to control data (5.1 $\pm 0.6 \mathrm{imp} / \mathrm{s}$ (52 recorded neurons), $P=0.3$; Figure 5).

Two weeks after the noise exposure, the spontaneous activity was significantly higher than in controls (8.9 \pm $1.3 \mathrm{imp} / \mathrm{s}$ (23 recorded neurons), $P=0.014$; Figure 5).

The original recordings did not show large differences in neuronal firing patterns at different time points after noise exposure. As indicated by the sample recordings (Figure 2), neuronal firing in general was quite regular without any certain changes in firing characteristics between the experimental or control groups. 


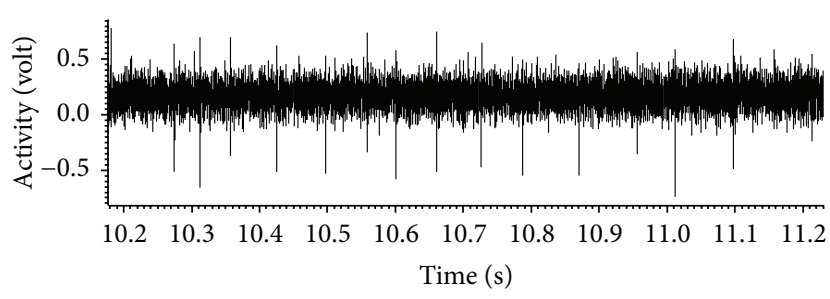

(a)

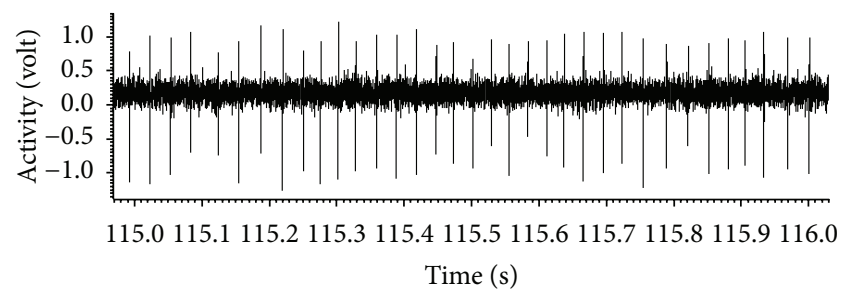

(b)

Figure 2: Example of filtered and amplified spike trains of DCN neurons from a control (a) and a 14-day group (b) animal, recorded with the spike2 software. Single units were classified using the template matching function of the spike2 software and mean spontaneous activity was calculated accordingly from the original recordings. Each diagram represents a one-second frame out of a 3-minute recording with the time in seconds on the $x$-axis and the activity in volt on the $y$-axis. The higher spike rate of the 14-day group neuron indicates the increase in spontaneous firing in the noise-exposed group two weeks after trauma.

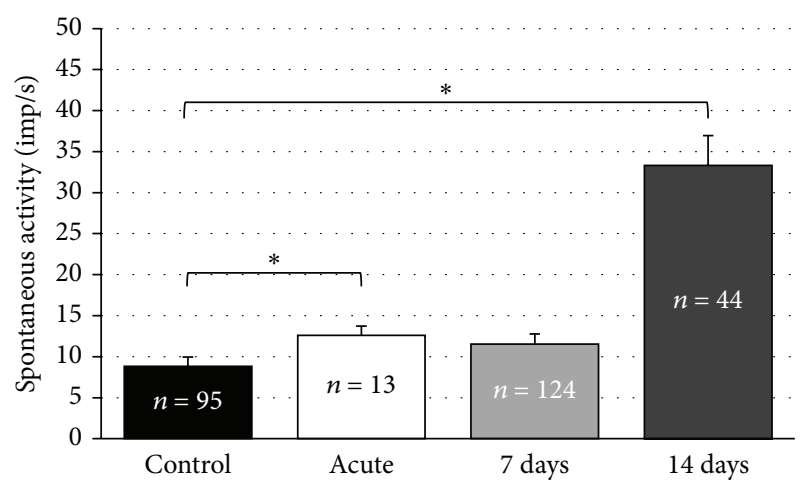

FIGURE 3: Spontaneous activities (mean \pm S.E.) of recorded neurons in the dorsal cochlear nucleus (DCN) of the control and noiseexposed groups. Numbers of recorded units for each subgroup are inserted inside of the columns. Asterisks indicate significant differences.



FIGURE 4: Spontaneous activities (mean \pm S.E.) of recorded neurons in the ventral cochlear nucleus (VCN) of the control and noiseexposed groups. Numbers of recorded units for each subgroup are inserted inside of the columns. Asterisks indicate significant differences.

Statistical analysis of tonotopically related spontaneous activity did not show any significant effects. Neither withingroup comparison between high- and low-frequency areas in each structure, nor comparison of matched subdivisions

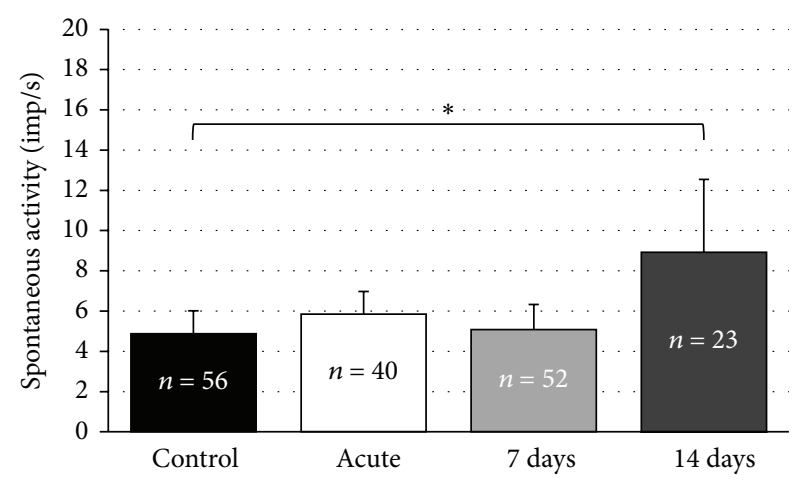

Figure 5: Spontaneous activities (mean \pm S.E.) of recorded neurons in the inferior colliculus (IC) of the control and noise-exposed groups. Numbers of recorded units for each subgroup are inserted inside of the columns. Asterisks indicate significant differences.

for each structure between experimental and control groups showed any frequency-related differences in changes of spontaneous activity. Thus, neuronal firing rates are supposed to show an equal distribution across the investigated auditory structures.

\section{Discussion}

The results of the present study demonstrate that acoustic overstimulation changes physiological properties of neurons within the lower central auditory pathway with a distinct temporal pattern. With our specific experimental design, we showed that the increases in neuronal firing rates are genuine, intrinsic cellular changes in isolated central auditory structures.

4.1. Hearing Thresholds. Previous studies showed that the applied noise paradigm leads to a significant elevation in auditory thresholds in all experimental groups with the highest shift in the acute group, followed by a significant recovery within one week (Figure 6, from [26]). However, hearing loss was still significantly increased after 7 days compared to controls and showed no further changes until 


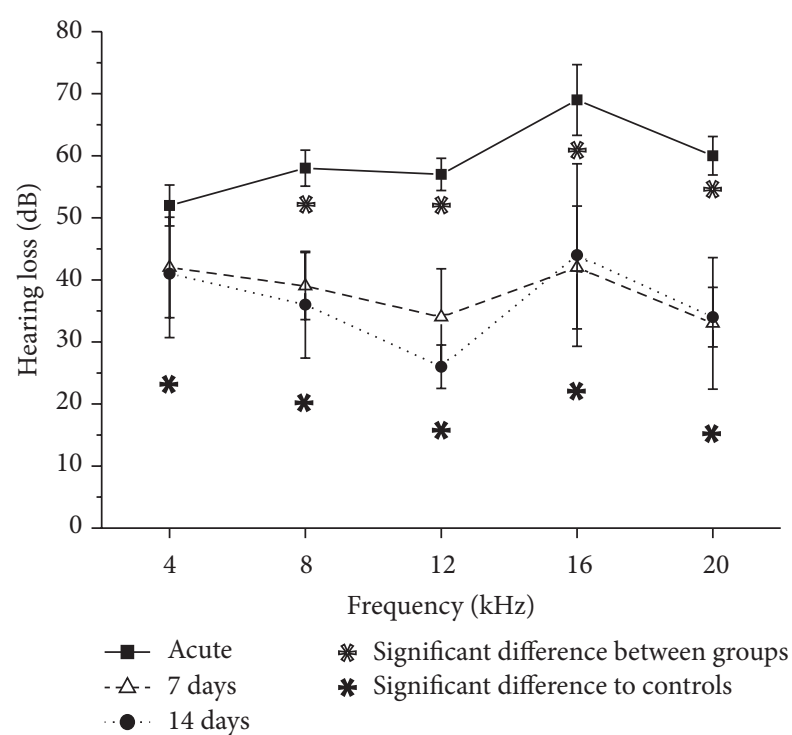

FIGURE 6: Threshold shift in dB SPL (mean \pm S.E.) of the auditory brainstem response (ABR) at different frequencies of noise-exposed mice (acute group: filled squares; 7-day group: open triangles; 14day group: filled circles). Filled asterisks show significant differences between treatment groups and controls. Open asterisks indicate significant recovery of auditory thresholds between the acute and 7-day group (from [26]).

day 14 , indicating that a permanent threshold shift (PTS) has developed.

Several studies have already reported that short-term processes within the cochlea (reversible and irreversible damage of sensory tissue) contribute to the acute threshold shift. A strong peripheral excitation by glutamate release reduces the energy and transmitter availability [1] and thereby inhibits further excitability. Similar mechanisms in central auditory structures might be responsible to some extent for the generation of sudden hearing loss.

4.2. Acute Effects. The above-mentioned idea is strongly supported by the present finding of a slight, but significant, elevation in spontaneous firing rate in the acute group at the first level of central auditory processing, that is, the cochlear nuclei. No differences were found in hierarchically higher structures. These experimental findings suggest that the applied noise trauma exerts a direct influence on the intrinsic, cellular network activity of the $\mathrm{CN}$ which in turn triggers several other pathophysiological events. This is also supported by a recent study of our group indicating a large increase in calcium-dependent activity in vivo immediately after acoustic overexposure [26].

An acute increase of peripheral activation of inner hair cells and spiral ganglion neurons [1] could induce a strong glutamate release at synapses between auditory nerve fibres and $\mathrm{CN}$ neurons, particularly at highly calcium-permeable, fast-transmitting AMPA receptors at the endbulb of Held's synapses. This leads to a fast and powerful long-lasting excitation [27]. These mechanisms might strongly contribute to acute hearing loss and result in short-term plastic changes [28], which is indicated by maintained excitatory transmitter release even after slice preparation. An immediate posttraumatic activity increase was also reported for the $\mathrm{CN}$ in vivo [29]. This overexcitation could induce neurodegenerative mechanisms such as apoptosis or even necrosis [30] as supported by recent findings on acute cell death in the $\mathrm{CN}$ after noise exposure $[10,31]$. An acute elevation in spontaneous activity in anaesthetized animals was also present in higher auditory structures, namely, the IC $[16,32]$. This effect was missing in our results and argues that IC hyperactivity at this time point is not based upon sustained physiological changes within this nucleus, but it is dependent on afferent (and perhaps efferent) connectivity. The absence of activity changes could also arise from protective mechanisms to reduce the direct impact on higher structures. Traumatic injury could be prevented by activation of inhibitory interneurons in the $\mathrm{CN}$ suppressing ascending neural transmission [33]. Moreover, rapid reduction in postsynaptic AMPA-receptor densities could avoid excitotoxicity. A similar mechanism was demonstrated in spiral ganglion neurons of the auditory nerve [34].

4.3. Late Effects. One week postexposure, a strong permanent hearing loss was established due to irreversible peripheral and central damage. In the cochlea, a loss of sensory tissue (hair cells and spiral ganglion neurons) has been observed $[35,36]$ resulting in reduced stimulus conduction to the auditory nuclei. Moreover, cell densities of involved brain areas are largely decreased at this time point due to apoptotic mechanisms $[9,10,31]$. The PTS findings at one week were confirmed by the measurements of the 14-day group.

Single-unit responses remained unchanged in all $\mathrm{CN}$ subdivisions as well as in the IC one week postexposure. However, when looking at the data of the animals investigated at 14 days after noise exposure, it turned out that intrinsic long-term hyperactivity in brain slices of central auditory structures was detected for the first time in the present study. Therefore, increase in neuronal spontaneous firing seems to develop within the period of two weeks, but not before day 7 after trauma. In general, mean spontaneous activity was much less expressed in the IC compared to the hierarchically lower $\mathrm{CN}$. This finding is in line with other studies investigating neuronal spontaneous firing in these structures before and after noise exposure [23]. Previous in vivo studies pointed out those long-lasting hyperactive disorders in central auditory structures after noise exposure, particularly within the lower auditory pathway, namely, the DCN and IC [14, 16, 37, 38]. These changes were shown to be induced within the first week after acoustic trauma and to gradually be increased in magnitude during the following days and months [22]. The DCN as well as an intact cochlea seems to be important for the maintenance of IC hyperactivity, as shown by in vivo lesioning studies [24, 37]. However, the intrinsic neuronal properties in both DCN and IC seem to be changed over time and are even present after isolation of the particular structures. It can be hypothesized that other neural connections, for example, descending projections from the auditory cortex, still have a significant impact on the reorganization of 
neurotransmission after noise trauma in the live animal [3941]. Nevertheless, the DCN plays a fundamental role as a relay for transmitting hyperactivity towards the IC [23], though our data suggest that the effects as reported for IC cells are not simply a passive response. This idea is also supported by Robertson et al. [42] who showed in guinea pigs that IC hyperactivity becomes independent of afferent neuronal input over time. Although the observed effects occur later compared to our findings, the involved mechanisms might be similar.

Brain slice recordings after a pure-tone noise exposure indicate that hyperactive disorders are partially caused by a lack of inhibition in the affected neurons [18, 43]. These studies showed reduced firing rates in $\mathrm{CN}$ and IC neurons of animals with noise-induced hearing loss which could be due to an increased postsynaptic GABA-receptor density. An increase in inhibitory glycine activity further contributes to the effect [44]. This causes a decline in spiking activity after slice preparation by removing the neuronal input to the relating auditory structures [45]. Differences to our present findings could be caused by the broad-band noise exposure in this study, followed by more widespread tissue damage with compensatory neuroplasticity, compared to narrowband or pure-tone sounds. This is confirmed by the equal distribution of neurons with specific firing rates across the dimensions of the investigated structures, accompanied by a significant overall shift in auditory thresholds in the noiseexposed animals across the entire tested frequency range. A broad-band noise trauma might therefore induce a different redistribution of posttraumatic excitatory and inhibitory neuronal properties, such as transmitter release, receptor densities, and synaptic strengths. Further, earlier studies were able to show that hyperactive disorders due to noise-induced hearing loss are often related to the frequency area of the detected hearing loss $[16,46]$. Thus, the absence of location(frequency-) specific hyperactivity in our data is possibly related to the induced extensive hearing loss, which has been detected in the present study.

Particularly in the DCN, an upregulation of cholinergic and glutamatergic neuronal input also seems to play an important role in generating hyperactivity [19, 47-49]. The shift in the balance of inhibition and excitation due to synaptic plasticity as compensatory response to neuronal deafferentiation seems to be the trigger of central hyperactivity [50]. The involved mechanisms could range from axonal sprouting and regrowth of synaptic connectivity in response to a loss of input [51-54] or homeostatic changes shifting the strengths of existing synapses due to input alterations [5557]. Neuronal hyperactivity might also develop in response to central degeneration to account for the deterioration of neural tissue. Recent studies of our group have demonstrated that cell death is particularly present within the first week after this noise trauma paradigm [10, 31]. Neuroplasticity might lead to profound changes in synaptic transmission during this development, but the present data support the assumption that spontaneous hyperactivity seems to be driven by an intact neural network at this time point. Although anatomical changes within the second week after noise trauma have not been investigated until now, it could be hypothesized from calcium activity monitoring that neuroplasticity and possibly neurodegeneration are largely reduced [26], and hyperactivity as a pathophysiological disorder might already be manifested within the neuronal structures of the lower central auditory pathway at this time.

\section{Conclusion}

The aim of the present study was to describe the temporal patterns of spontaneous, neuronal single-unit activity upon noise-induced hearing loss in brain slices of the dorsal and ventral cochlear nucleus as well as the inferior colliculus. This experimental procedure allows measuring the intrinsic acute and long-term activity changes in structures of the lower auditory pathway isolated from afferent or efferent neuronal input. An increased spontaneous activity seems to develop within two weeks, but not before day 7 after acoustic trauma. Altogether, the present study provides a deeper insight into the complex alterations in neuronal physiological properties within the adult central auditory system after a single noise trauma. The results should contribute to a better understanding of central mechanisms in acute and permanent noiseinduced hearing loss.

\section{Conflict of Interests}

The authors declare that there is no conflict of interests regarding the publication of this paper.

\section{References}

[1] D. Henderson, E. C. Bielefeld, K. C. Harris, and B. H. Hu, "The role of oxidative stress in noise-induced hearing loss," Ear and Hearing, vol. 27, no. 1, pp. 1-19, 2006.

[2] A. S. Nordmann, B. A. Bohne, and G. W. Harding, "Histopathological differences between temporary and permanent threshold shift," Hearing Research, vol. 139, no. 1-2, pp. 13-30, 2000.

[3] E. C. Kane, "Patterns of degeneration in the caudal cochlear nucleus of the cat after cochlear ablation," The Anatomical Record, vol. 179, no. 1, pp. 67-91, 1974.

[4] M. Jean-Baptiste and D. K. Morest, "Transneuronal changes of synaptic endings and nuclear chromatin in the trapezoid body following cochlear ablations in cats," Journal of Comparative Neurology, vol. 162, no. 1, pp. 111-133, 1975.

[5] J. Kim, D. K. Morest, and B. A. Bohne, "Degeneration of axons in the brainstem of the chinchilla after auditory overstimulation," Hearing Research, vol. 103, no. 1-2, pp. 169-191, 1997.

[6] A. A. Aarnisalo, U. Pirvola, X. Q. Liang, J. Miller, and J. Ylikoski, "Apoptosis in auditory brainstem neurons after a severe noise trauma of the organ of corti: intracochlear GDNF treatment reduces the number of apoptotic cells," $O R L$, vol. 62, no. 6, pp. 330-334, 2000.

[7] S. K. Suneja, S. J. Potashner, and C. G. Benson, "Plastic changes in glycine and GABA release and uptake in adult brain stem auditory nuclei after unilateral middle ear ossicle removal and cochlear ablation," Experimental Neurology, vol. 151, no. 2, pp. 273-288, 1998.

[8] S. K. Suneja, S. J. Potashner, and C. G. Benson, "AMPA receptor binding in adult guinea pig brain stem auditory nuclei after 
unilateral cochlear ablation," Experimental Neurology, vol. 165, no. 2, pp. 355-369, 2000.

[9] D. Basta, B. Tzschentke, and A. Ernst, "Noise-induced cell death in the mouse medial geniculate body and primary auditory cortex," Neuroscience Letters, vol. 381, no. 1-2, pp. 199-204, 2005.

[10] M. Gröschel, R. Götze, A. Ernst, and D. Basta, "Differential impact of temporary and permanent noise-induced hearing loss on neuronal cell density in the mouse central auditory pathway," Journal of Neurotrauma, vol. 27, no. 8, pp. 1499-1507, 2010.

[11] P. Dallos, D. Harris, O. Ozdamar, and A. Ryan, "Behavioral, compound action potential, and single unit thresholds: relationship in normal and abnormal ears," Journal of the Acoustical Society of America, vol. 64, no. 1, pp. 151-157, 1978.

[12] R. J. Salvi, D. Ding, J. Wang, and H. Y. Jiang, "A review of the effects of selective inner hair cell lesions on distortion product otoacoustic emissions, cochlear function and auditory evoked potentials," Noise and Health, vol. 2, no. 6, pp. 9-25, 2000.

[13] M. C. Liberman and N. Y. Kiang, "Acoustic trauma in cats. Cochlear pathology and auditory-nerve activity," Acta OtoLaryngologica, Supplement, vol. 358, pp. 1-63, 1978.

[14] J. A. Kaltenbach, D. A. Godfrey, J. B. Neumann, D. L. McCaslin, C. E. Afman, and J. Zhang, "Changes in spontaneous neural activity in the dorsal cochlear nucleus following exposure to intense sound: relation to threshold shift," Hearing Research, vol. 124, no. 1-2, pp. 78-84, 1998.

[15] A. J. Noreña and J. J. Eggermont, "Changes in spontaneous neural activity immediately after an acoustic trauma: implications for neural correlates of tinnitus," Hearing Research, vol. 183, no. 1-2, pp. 137-153, 2003.

[16] W. D. Ma, H. Hidaka, and B. J. May, "Spontaneous activity in the inferior colliculus of CBA/J mice after manipulations that induce tinnitus," Hearing Research, vol. 212, no. 1-2, pp. 9-21, 2006.

[17] R. J. Salvi, S. S. Saunders, M. A. Gratton, S. Arehole, and N. Powers, "Enhanced evoked response amplitudes in the inferior colliculus of the chinchilla following acoustic trauma," Hearing Research, vol. 50, no. 1-2, pp. 245-258, 1990.

[18] D. Basta and A. Ernst, "Erratum to 'Noise-induced changes of neuronal spontaneous activity in mice inferior colliculus brain slices"' Neuroscience Letters, vol. 374, no. 1, pp. 74-79, 2005.

[19] J. A. Kaltenbach and J. Zhang, "Intense sound-induced plasticity in the dorsal cochlear nucleus of rats: evidence for cholinergic receptor upregulation," Hearing Research, vol. 226, no. 1-2, pp. 232-243, 2007.

[20] S. A. Michler and R. B. Illing, "Acoustic trauma induces reemergence of the growth- and plasticity-associated protein GAP43 in the rat auditory brainstem," The Journal of Comparative Neurology, vol. 451, pp. 250-266, 2002.

[21] J. W. House and D. E. Brackmann, "Tinnitus: surgical treatment.," Ciba Foundation Symposium, vol. 85, pp. 204-216, 1981.

[22] J. A. Kaltenbach, J. Zhang, and C. E. Afman, "Plasticity of spontaneous neural activity in the dorsal cochlear nucleus after intense sound exposure," Hearing Research, vol. 147, pp. 282$292,2000$.

[23] N. F. Manzoor, Y. Gao, F. Licari, and J. A. Kaltenbach, "Comparison and contrast of noise-induced hyperactivity in the dorsal cochlear nucleus and inferior colliculus," Hearing Research, vol. 295, pp. 114-123, 2013.

[24] N. F. Manzoor, F. G. Licari, M. Klapchar et al., "Noiseinduced hyperactivity in the inferior colliculus: its relationship with hyperactivity in the dorsal cochlear nucleus," Journal of Neurophysiology, vol. 108, pp. 976-988, 2012.
[25] G. Paxinos and K. B. J. Franklin, The Mouse Brain in Stereotaxic Coordinates, Elsevier Science \& Technology, Oxford, UK, 2001.

[26] M. Gröschel, S. Müller, R. Götze, A. Ernst, and D. Basta, "The possible impact of noise-induced $\mathrm{Ca}^{2+}$-dependent activity in the central auditory pathway: a manganese-enhanced MRI study," NeuroImage, vol. 57, pp. 190-197, 2011.

[27] Y. Wang and P. B. Manis, "Short-term synaptic depression and recovery at the mature mammalian endbulb of Held synapse in mice," Journal of Neurophysiology, vol. 100, no. 3, pp. 1255-1264, 2008.

[28] J. Xu, L. He, and L. G. Wu, "Role of $\mathrm{Ca}^{2+}$ channels in short-term synaptic plasticity," Current Opinion in Neurobiology, vol. 17, pp. 352-359, 2007.

[29] F. A. Boettcher and R. J. Salvi, "Functional changes in the ventral cochlear nucleus following acute acoustic overstimulation," The Journal of the Acoustical Society of America, vol. 94, pp. 21232134, 1993.

[30] E. Salińska, W. Danysz, and J. W. Łazarewicz, "The role of excitotoxicity in neurodegeneration," Folia Neuropathologica, vol. 43, no. 4, pp. 322-339, 2005.

[31] A. Coordes, M. Gröschel, A. Ernst, and D. Basta, "Apoptotic cascades in the central auditory pathway after noise exposure," Journal of Neurotrauma, vol. 29, no. 6, pp. 1249-1254, 2012.

[32] J. Wang, R. J. Salvi, and N. Powers, "Plasticity of response properties of inferior colliculus neurons following acute cochlear damage," Journal of Neurophysiology, vol. 75, no. 1, pp. 171-183, 1996.

[33] A. F. Ryan, G. A. Axelsson, and N. K. Woolf, "Central auditory metabolic activity induced by intense noise exposure," Hearing Research, vol. 61, no. 1-2, pp. 24-30, 1992.

[34] Z. Chen, M. Peppi, S. G. Kujawa, and W. F. Sewell, "Regulated expression of surface AMPA receptors reduces excitotoxicity in auditory neurons," Journal of Neurophysiology, vol. 102, pp. 1152$1159,2009$.

[35] R. Pujol and J. L. Puel, "Excitotoxicity, synaptic repair, and functional recovery in the mammalian cochlea: a review of recent findings," Annals of the New York Academy of Sciences, vol. 884, pp. 249-254, 1999.

[36] B. H. Hu, D. Henderson, and T. M. Nicotera, "Involvement of apoptosis in progression of cochlear lesion following exposure to intense noise," Hearing Research, vol. 166, no. 1-2, pp. 62-71, 2002.

[37] W. H. Mulders and D. Robertson, "Hyperactivity in the auditory midbrain after acoustic trauma: dependence on cochlear activity," Neuroscience, vol. 164, pp. 733-746, 2009.

[38] W. L. Ma and E. D. Young, "Dorsal cochlear nucleus response properties following acoustic trauma: response maps and spontaneous activity," Hearing Research, vol. 216-217, pp. 176-188, 2006.

[39] S. Seki and J. J. Eggermont, "Changes in spontaneous firing rate and neural synchrony in cat primary auditory cortex after localized tone-induced hearing loss," Hearing Research, vol. 180, no. 1-2, pp. 28-38, 2003.

[40] N. E. Meltzer and D. K. Ryugo, "Projections from auditory cortex to cochlear nucleus: a comparative analysis of rat and mouse," The Anatomical Record A, Discoveries in Molecular, Cellular, and Evolutionary Biology, vol. 288, no. 4, pp. 397-408, 2006.

[41] V. M. Bajo, F. R. Nodal, J. K. Bizley, D. R. Moore, and A. J. King, "The ferret auditory cortex: descending projections to the inferior colliculus," Cerebral Cortex, vol. 17, pp. 475-491, 2007. 
[42] D. Robertson, C. Bester, D. Vogler, and W. H. Mulders, "Spontaneous hyperactivity in the auditory midbrain: relationship to afferent input," Hearing Research, vol. 295, pp. 124-129, 2013.

[43] H. Chang, K. Chen, J. A. Kaltenbach, J. Zhang, and D. A. Godfrey, "Effects of acoustic trauma on dorsal cochlear nucleus neuron activity in slices," Hearing Research, vol. 164, pp. 59-68, 2002.

[44] H. Wang, T. J. Brozoski, J. G. Turner et al., "Plasticity at glycinergic synapses in dorsal cochlear nucleus of rats with behavioral evidence of tinnitus," Neuroscience, vol. 164, no. 2, pp. 747-759, 2009.

[45] A. G. Holt, M. Asako, C. A. Lomax et al., "Deafness-related plasticity in the inferior colliculus: gene expression profiling following removal of peripheral activity," Journal of Neurochemistry, vol. 93, pp. 1069-1086, 2005.

[46] J. A. Kaltenbach, J. Zhang, and P. Finlayson, "Tinnitus as a plastic phenomenon and its possible neural underpinnings in the dorsal cochlear nucleus," Hearing Research, vol. 206, pp. 200-226, 2005.

[47] N. F. Manzoor, G. Chen, and J. A. Kaltenbach, "Suppression of noise-induced hyperactivity in the dorsal cochlear nucleus following application of the cholinergic agonist, carbachol," Brain Research, vol. 1523, pp. 28-36, 2013.

[48] D. A. Godfrey, J. A. Kaltenbach, K. Chen, and O. Ilyas, "Choline acetyltransferase activity in the hamster central auditory system and long-term effects of intense tone exposure," Journal of Neuroscience Research, vol. 91, pp. 987-996, 2013.

[49] C. Zeng, N. Nannapaneni, J. Zhou, L. F. Hughes, and S. Shore, "Cochlear damage changes the distribution of vesicular glutamate transporters associated with auditory and nonauditory inputs to the cochlear nucleus," The Journal of Neuroscience, vol. 29, no. 13, pp. 4210-4217, 2009.

[50] L. E. Roberts, J. J. Eggermont, D. M. Caspary, S. E. Shore, J. R. Melcher, and J. A. Kaltenbach, "Ringing ears: the neuroscience of tinnitus," The Journal of Neuroscience, vol. 30, pp. 1497214979, 2010.

[51] C. G. Benson, J. S. Gross, S. K. Suneja, and S. J. Potashner, "Synaptophysin immunoreactivity in the cochlear nucleus after unilateral cochlear or ossicular removal," Synapse, vol. 25, pp. 243-257, 1997.

[52] J. J. Kim, J. Gross, D. K. Morest, and S. J. Potashner, “Quantitative study of degeneration and new growth of axons and synaptic endings in the chinchilla cochlear nucleus after acoustic overstimulation," Journal of Neuroscience Research, vol. 77, no. 6, pp. 829-842, 2004.

[53] J. J. Kim, J. Gross, S. J. Potashner, and D. K. Morest, "Fine structure of long-term changes in the cochlear nucleus after acoustic overstimulation: chronic degeneration and new growth of synaptic endings," Journal of Neuroscience Research, vol. 77, no. 6, pp. 817-828, 2004.

[54] M. Bilak, J. Kim, S. J. Potashner, B. A. Bohne, and D. K. Morest, "New growth of axons in the cochlear nucleus of adult chinchillas after acoustic trauma," Experimental Neurology, vol. 147, pp. 256-268, 1997.

[55] G. G. Turrigiano, "Homeostatic plasticity in neuronal networks: the more things change, the more they stay the same," Trends in Neurosciences, vol. 22, no. 5, pp. 221-227, 1999.

[56] G. G. Turrigiano, K. R. Leslie, N. S. Desai, L. C. Rutherford, and S. B. Nelson, "Activity-dependent scaling of quantal amplitude in neocortical neurons," Nature, vol. 391, pp. 892-896, 1998.
[57] R. Schaette and R. Kempter, "Development of tinnitus-related neuronal hyperactivity through homeostatic plasticity after hearing loss: a computational model," European Journal of Neuroscience, vol. 23, pp. 3124-3138, 2006. 


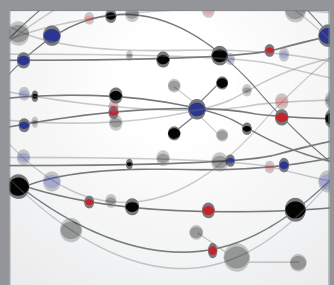

The Scientific World Journal
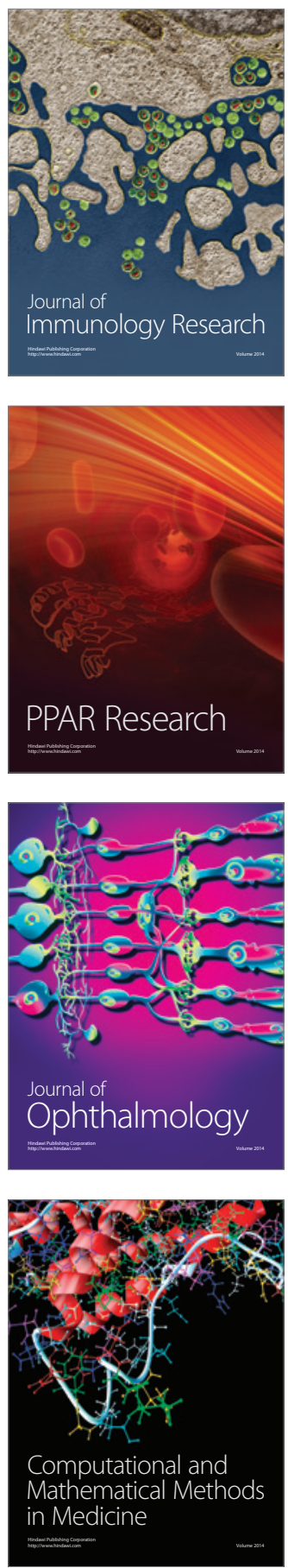

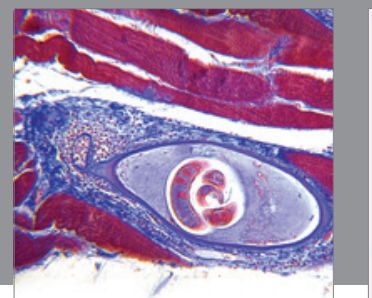

Gastroenterology

Research and Practice
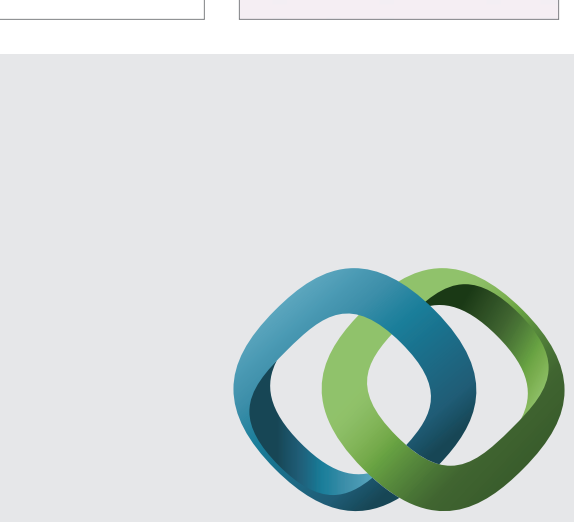

\section{Hindawi}

Submit your manuscripts at

http://www.hindawi.com
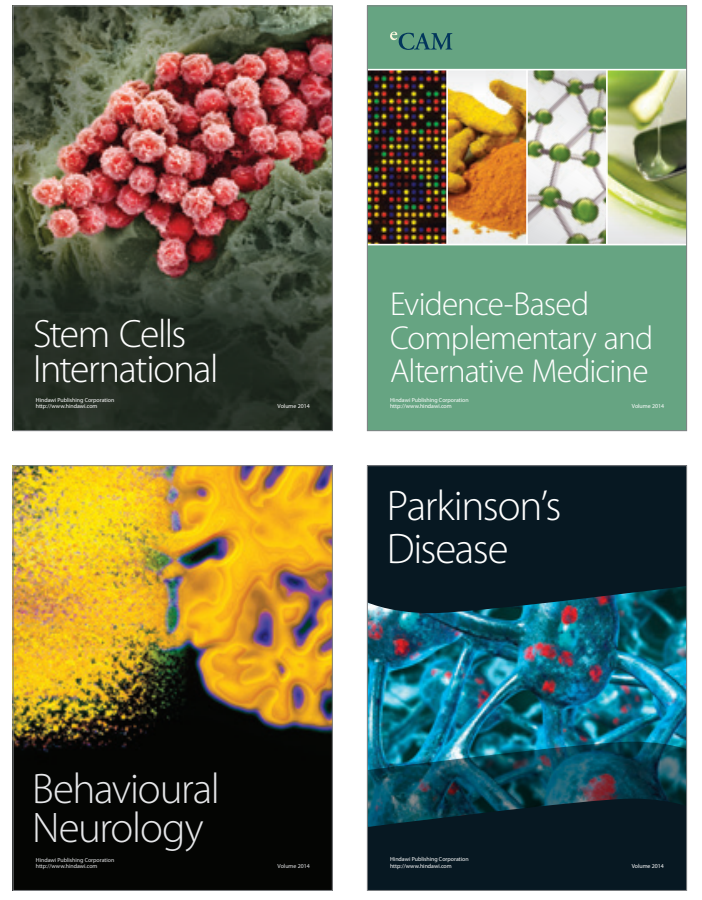
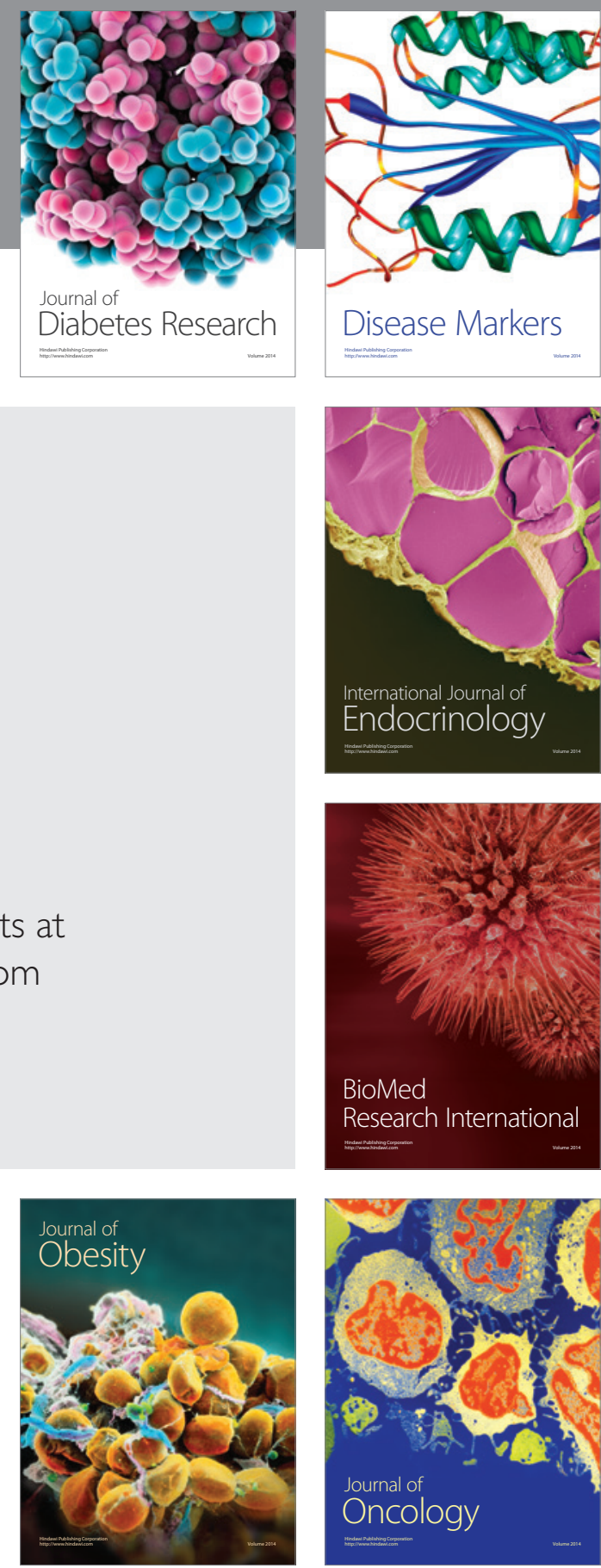

Disease Markers
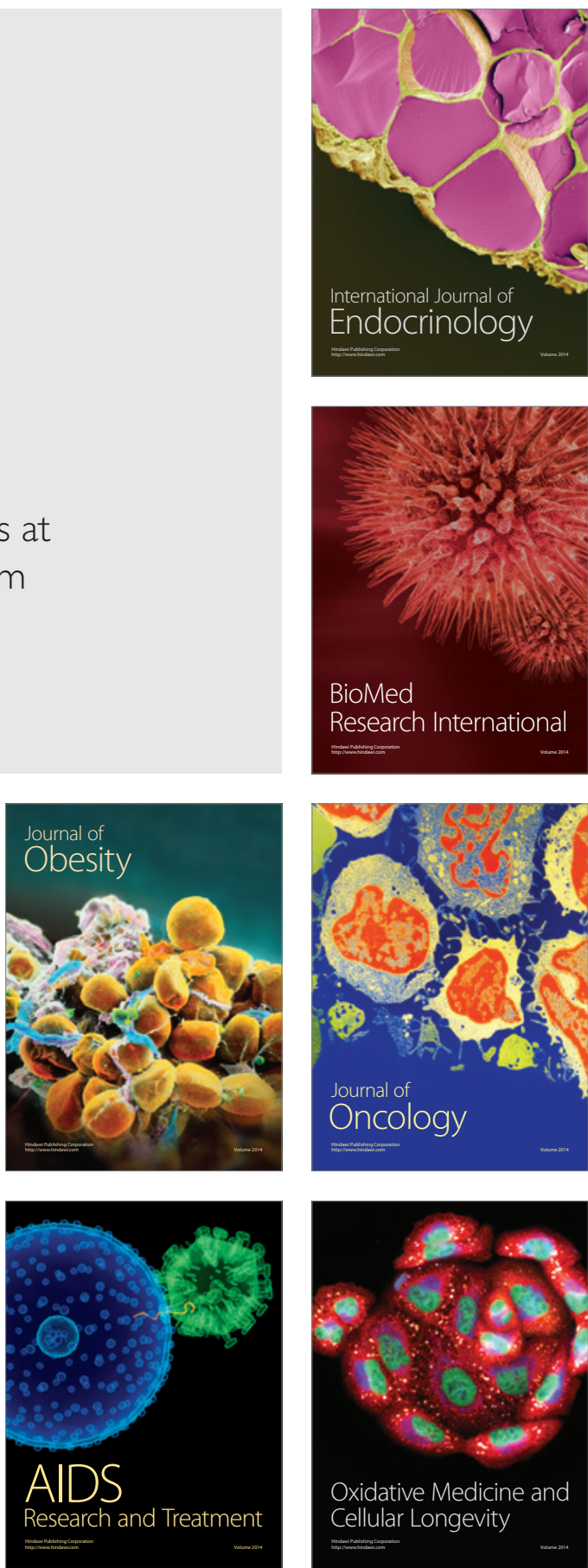